\title{
THE EVALUATION OF ISCHEMIA MODIFIED ALBUMIN AND ADROPIN LEVELS IN PATIENTS WITH PULMONARY EMBOLISM
}

\section{Said Sami Erdem ${ }^{1}$, Kamile Yücel ${ }^{2}$, Yavuz Turgut Gederet, Ercan Kurtipek $^{3}$, Taha Tahir Bekci ${ }^{3}$, Hüseyin Kurku ${ }^{1}$}

${ }^{1}$ Konya Education and Research Hospital, Department of Biochemistry, Konya, Turkey

2 KTO Karatay University, School of Health Sciences, Department of Medical Biochemistry, Konya, Turkey

${ }^{3}$ Konya Education and Research Hospital, Department of Pulmonary Medicine, Konya, Turkey

Address for Correspondence: Kamile Yucel, E-mail: kamile_yucel@hotmail.com Received: 19.01.2021; Accepted: 06.04.2021; Available Online Date: 27.05.2021

CCopyright 2021 by Dokuz Eylül University, Institute of Health Sciences - Available online at https://dergipark.org.tr/en/pub/jbachs

Cite this article as: Yucel K, Erdem SS, Gederet YT, Kurtipek E, Bekci TT, Kurku H. The Evaluation of Ischemia Modified Albumin and Adropin Levels in Patients with Pulmonary Embolism. J Basic Clin Health Sci 2021; 2: 59-64.

\begin{abstract}
Purpose: Pulmonary embolism (PE) is a serious clinical condition and difficult to be diagnosed. The aim of the present study was to evaluate plasma adropin and ischemia-modified albumin (IMA) levels in the patients with PE.

Methods: The study consisted of 109 participants, including 50 patients with PE and 59 healthy individuals. The patients diagnosed with PE through computed tomography pulmonary angiography (CTPA) were included into the study. Plasma adropin and IMA concentrations were measured by an enzyme-linked immunosorbent assay kit.
\end{abstract}

Results: The patients with PE had higher plasma adropin (607.5 $\pm 205.1 \mathrm{ng} / \mathrm{mL})$ and lower IMA $(2128.6 \pm 801.8 \mathrm{ng} / \mathrm{mL})$ levels, compared with those in the controls $(505.5 \pm 231.3 \mathrm{ng} / \mathrm{mL}$ and $2466.9 \pm 1044.9 \mathrm{ng} / \mathrm{mL}$, respectively); however, the difference was not significant. In the study, we found a weak and negative correlation between adropin and IMA levels in the patients with PE $(p=0.020, r=-$ 0.348).

Conclusions: In our study among the patients with PE, there was no significant difference between adropin and IMA levels, although plasma atropin concentrations were high, and IMA concentrations were low. So, we consider that both IMA and adropin are inappropriate markers for the diagnosis of PE.

Keywords: Adropin, ischemia-modified albumin, oxidative stress, pulmonary embolism, receiver operating characteristics

\section{INTRODUCTION}

Pulmonary embolism (PE) is a serious clinical condition, resulting from the obstruction of the pulmonary arteries or related branches due to the thrombus caused by leg deep veins $(1,2)$. Circulatory instability, hypoperfusion, hypoxia and ischemia occur due to the failure of pulmonary circulation caused by pulmonary embolism. The etiological causes of PE are evaluated into two parts as inherited and acquired. Among the etiological mechanisms, vascular endothelial damage, hypercoagulability and stasis can be assessed as the primary causes. Although the mortality rate in patients with $\mathrm{PE}$ is high, the rate is decreased among the cases treated appropriately and promptly, and with the help of accurate diagnosis (2-4). The first factor to help physicians diagnose PE is the clinical findings of PE displayed by the patient and suspected by the 
clinician $(3,5)$. The number of publications related to clinical practice has been increased according to the guidelines published by the European Society of Cardiology (6). However, the diagnosis of venous thromboembolism and PE still remains as a challenge because of non-specific conditions, and because the symptoms of the accompanying diseases can mask the clinical picture $(7,8)$. The initial assessment and suspecting at pre-diagnosis by the healthcare worker are of critical importance to identify $P E$, and computed tomography pulmonary angiography (CTPA) is also the most sensitive and accurate diagnostic method for highly probable cases. Since none of the laboratory tests can achieve a definitive diagnosis of $P E$, healthcare professionals must rely on the pulmonary angiography and CTPA $(9,10)$. In order to reduce the number of complex imaging techniques, reliable, simple and novel techniques are needed to diagnose PE.

Adropin is a 76-amino-acid-including hormone, and expressed in the brain and liver by the energy homeostasis-associated gene $(11,12)$. Based on literature, adropin has also been associated with multiple diseases, including diabetes mellitus (DM), myocardial infarction, coronary atherosclerosis, obesity and non-alcoholic fatty liver disease $(13,14)$. The role and effects of adropin on glucose, insulin sensitivity and lipid homeostasis were first described in 2008 by Kumar et al. and later in 2014 by Aydin $(15,16)$. Although the mechanism of adropin still remains unelucidated, it is known that adropin has several effects on cardiovascular system, and metabolic and vascular homeostasis $(17,18)$. Endothelial cells regulate vascular homeostasis, and any change in the functional and morphological structure of the endothelium brings about the loss of normal physiological processes, known as endothelial dysfunction $(19,20)$. Low levels of adropin are considered to cause endothelial damage and dysfunction (17). The protective function of adropin in the endothelium has been demonstrated in a study performed by Lovren et al. in 2010 (19).

The N-terminal end of amino acid in albumin has a capacity to bind to heavy metals, such as copper, nickel and cobalt (21). In such pathological conditions as energy-induced membrane destruction, exposure to free iron and copper, acidosis and hypoxia, and a free radical damage developing in ischemia, the binding capacity of metals to the $\mathrm{N}$-terminal domain of albumin is decreased $(18,22)$. Ischemia-modified albumin (IMA) is formed by albumin modification caused by reactive oxygen derivatives due to ischemia. IMA is used as a sensitive biochemical marker of ischemia and oxidative stress caused by tissue hypoxia $(23,24)$. In previous studies, it was reported that IMA levels increase among patients with ischemia, acute rheumatic fever, hepatic failure, chronic obstructive pulmonary disease (COPD) and cerebro-vascular disease. The common feature of all these conditions is that such disorders present with increased reactive oxygen radicals $(22,25)$. Therefore, the patients with lower levels of IMA can be categorized as low-risk groups, and thereby lower levels of IMA may help provide major cost-effective outcomes. In different studies where IMA levels were measured in those with PE, different outcomes were reported. While some studies showed IMA levels to be increased, others stated that no changes were obsereved at the levels of IMA $(26,27)$. Based on literature, no studies have been encountered, investigating adropine levels in PE patients. In the present study, it was aimed to evaluate the levels of adropin and IMA in PE patients requiring to be diagnosed and intervened immediately. We consider that the rapid diagnosis of $\mathrm{PE}$ through a non-invasive biochemical marker will significantly reduce the mortality and morbidity rates, and that our study will contribute to literature. Therefore, our study is a preliminary study aiming to investigate whether adropin and IMA can be used in the diagnosis, exclusion and follow-up of PE.

\section{METHODS \\ Study population}

Fifty patients diagnosed with PE in the Department of Pulmonary Medicine of Konya Education and Research Hospital between January 2017 and January 2018, and 59 healthy volunteers were included into the study. The samples obtained from the patients and controls during routine procedures were planned to be used with no further invasive procedure being undertaken. The control group consisted of individuals without DM, hypertension, COPD, or obesity. As exclusion criteria, those with the history of CAD, abnormal plasma albumin levels leading to masking actual of IMA levels, chronic renal failure, significant valvular heart disease, history of acute and/or chronic infections, malignancies, treated with any steroidal and non-steroidal anti-inflammatory drug and not accepting to sign the consent were excluded out of the study. 
Table 1. Plasma adropin and IMA levels of patients with PE and controls

\begin{tabular}{lllc}
\hline Characteristics & $\begin{array}{l}\mathrm{PE}(\mathrm{n}=50)(\text { median } \pm \text { SD }) \\
(\min -\mathrm{max})\end{array}$ & $\begin{array}{l}\text { Controls }(\mathrm{n}=59)(\text { median } \pm \text { SD }) \\
(\min -\max )\end{array}$ & $\mathrm{p}$ value \\
\hline Adropin $(\mathrm{ng} / \mathrm{mL})$ & $607.5 \pm 205.1(337.5-1098.5)$ & $500.5 \pm 231.3(150.5-1188.6)$ & 0.178 \\
IMA $(\mathrm{ng} / \mathrm{mL})$ & $2128.6 \pm 801.8(405.1-3831.9)$ & $2466.9 \pm 1044.9(839.2-5090.1)$ & 0.141 \\
\hline
\end{tabular}

*Significant as to $p<0.05$ level.

\section{Biochemical analyses}

Our study was performed with blood samples taken immediately after the hospitalization of patients diagnosed with PE. The drawn blood samples were centrifuged at 3.000xrpm for $15 \mathrm{~min}$ and stored at -80 ${ }^{\circ} \mathrm{C}$ until the enzyme-linked immunosorbent assay (ELISA) ran. Adropin and IMA levels were measured simultaneously on the same samples. Adropin concentrations were measured with the ELISA kit (Cat. No. 201-12-3107 Sunred Biological Technology Co., Shanghai, PRC) in light of the manufacturer's manual. The coefficients of variation (CVs) were $<9 \%$ for intra-assay and $<11 \%$ for inter-assay; the sensitivity was $4.735 \mathrm{pg} / \mathrm{mL}$, and the assay range was found as 5-1.000 pg/mL. Plasma IMA concentrations were measured via the ELISA kit (Cat. No. 201-121173 Sunred Biological Technology Co., Shanghai, PRC) according to the manufacturer's manual. For this analysis, the sensitivity was found as 2.226 $\mathrm{ng} / \mathrm{mL}$, while the assay range was $3-600 \mathrm{ng} / \mathrm{mL}$.

\section{Statistical analyses}

All statistical analyses were performed using Statistical Package for the Social Sciences package (SPSS, version 18.0 for Windows, SPSS INC., Chicago, IL., USA). The data obtained were expressed as numbers, percentages, and mean \pm standard deviation (SD). The KolmogorovSmirnov and the Shapiro-Wilk tests were conducted to evaluate whether the data conformed to the normal distribution. The normality test was performed, and the parametric tests were used for normally distributed variables $(p>0.05)$, while the nonparametric tests were utilized for not normally distributed variables $(p<0.05)$. The independent sampling $t$ test was used for comparing the continuous variables with the normal distributions between the groups. The Mann-Whitney $U$ test was used to compare intergroup non-parametric variables. The Pearson test was used for the correlation of parametric data. The association between the continuous variables was analyzed using the non-parametric Spearman correlation test in the cases where the data were not consistent with the normal distributions. The receiver operating characteristics (ROC) curve was used to determine the sensitivity and specificity of adropin and IMA, and the cut-off values in predicting PE. A $p<0.05$ was considered to indicate statistical significance.

\section{RESULTS}

The plasma adropin and IMA levels of PE patients and controls are shown in Table 1. The patients with PE had higher plasma adropin levels (607.5 \pm 205.1 $\mathrm{ng} / \mathrm{mL})$ and lower IMA levels $(2128.6 \pm 801.8 \mathrm{ng} / \mathrm{mL})$, compared with those of controls $(500.5 \pm 231.3$ and $2466.9 \pm 1044.9$, respectively), but the difference was not significant $(p=0.178, p=0.141$, respectively). A weak and negative correlation was found between the adropin and IMA levels in patients with PE $(p=0.020$, $r=-0.348$ ).

On diagnostic performance analysis, the ROC curve demonstrated the cut-off value of adropin as 450 or higher, the sensitivity level as $81.8 \%$, the specificity as $36.4 \%$, positive predictive value as $56.3 \%$ and negative predictive value as $66.7 \%$ in terms of $\mathrm{PE}$ (area under curve $(\mathrm{AUC})=0.583 ; 95 \%$ confidence interval $(\mathrm{Cl})=0.463-0.704)$ ). On the other hand, the cut-off value of IMA was found as 1761 , the sensitivity level as $72.7 \%$, the specificity as $29.5 \%$, positive predictive value as $50.8 \%$, while the negative predictive value was observed as $52.0 \%$ in terms of PE (AUC=0.436; 95\% Cl=0.314-0.559) (Figure).

\section{DISCUSSION}

In this study, no statistically significant difference was found between the patient and control groups in terms of adropin levels. A weak and negative correlation was found between adropin and IMA levels in patients with PE ( $r=-0.348)$, as a significant finding. To the best of our knowledge, there is no study in literature focusing on the relation between adropin and PE, as parallel to our results. Initially, we considered that endothelial dysfunction developing in PE would have 
an influence on adropin, but we noticed in later stages that limited number of study population, insufficient information in other studies were the limitations preventing us to elucidate why adropin levels were higher in PE patients. We have no information about whether other unknown factors, except for the endothelial dysfunction, play a role in the etiopathogenesis of our PE patients.

IMA is accepted as a marker of oxidative stress. In the presence of acute clots, an increase is seen at Ddimer levels in plasma due to the simultaneous activation of the fibrinolytic system by coagulation. Although the sensitivity of the D-dimer test is high, its specificity is low $(24,27)$. In several studies, it was reported that IMA is a more sensitive test, compared with D-dimer, in the diagnosis of PE $(28,29)$. In another study performed with 60 individuals (including 30 patients and 30 controls), Turedi et al. reported that IMA levels were markedly higher in $97.7 \%$ of PE patients (29). As parallel to the findings reported by Turedi et al., plasma IMA levels were demonstrated to be significantly higher in patients with $\mathrm{PE}$ than healthy controls in the study by Kaya et al. $(p=0.001)(27)$. Unlike the findings in these studies, although plasma IMA levels were lower in patients with $\mathrm{PE}$ than those among controls, no significant difference was found in our study $(p=$ 0.141). In the study by Turedi et al. (2008), however, the AUC was calculated for IMA as 0.889 in patients with PE (29). Even so, since mean IMA levels and overall distribution within patient group had a downward tendency compared to controls in our study, the AUC for IMA was found to be $<0.5$ in the ROC analysis. The number of studies showing the diagnostic value of IMA in PE patients is limited. In several studies, either increased or unchanged IMA levels have been reported in patients with PE (27-29). Compared the plasma IMA levels between PE patients and healthy subjects, no statistically significant difference was found in our study. Although no difference was found in terms of IMA levels in our PE patients, we consider that the reasons why the levels were reported significantly higher in previous studies were the methodological alterations and the number of participants.

While the albumin levels of the patients ranging between $3.5-5.5 \mathrm{mg} / \mathrm{dL}$ were evaluated in other studies, all patients with PE were evaluated in our study without taking the range into consideration. Considering also the underlying diseases in PE patients, hypoalbuminemia is an expected clinical condition. Therefore, we consider that hypoalbuminemic patients should not be excluded out of the study. In some studies, IMA levels are given as absorbance values (22, 27-29). However, IMA levels were measured as $\mathrm{ng} / \mathrm{mL}$. As well as hypoalbuminemia, as another reason for the inconsistency between the findings reported in other studies $(28,29)$ and those in our study, the culprits may be the presence of underlying diseases in patients with $\mathrm{PE}$, such as malignancies, infections and vascular diseases. Such ailments are known to cause a decrease in albumin levels and an increase in oxidative stress.

\section{CONCLUSION}

It is important to use a marker for diagnosing PE and showing the changes according to the size of ischemia immediately after ischemia-induced events. Based on our study findings, we consider that adropin cannot be used as a reliable marker in the diagnosis of PE, and IMA provides only limited benefits, and that further comprehensive studies are required to demonstrate whether IMA can be used as a marker to diagnose PE by evaluating the albumin unit of ABSU/gr in terms of plasma albumin levels.

\section{Limitations of the Study}

In our study, the first limitation was the small sample size. Secondly, the study was conducted in a singlecenter. In addition, no procedures were performed to assess the antioxidant parameters, such as lipid hydroperoxides, as to patients' daily diet although those taking vitamin supplements were excluded. Although there are few studies evaluating the effects of IMA on PE, no studies investigating the effects of adropin on PE were found, and therefore, we could find no sources to compare our findings.

Conflicts of interest : The authors declare no conflict of interests. No financial support was granted to the present study by any commercial organization. The study was also performed with respect to the recommendations via the 1960 Declaration of Helsinki and its following amendments. An ethical approval was also obtained from the Health Sciences Institute of Selcuk University, Konya, Turkey (Ethics Committee No: 2016/16, date: 12/01/2016).

Peer-review: Externally peer-reviewed.

\section{REFERENCES}

1. Root CW, Dudzinski DM, Zakhary B, Friedman OA, Sista AK, et al. Multidisciplinary approach to the management of pulmonary embolism 
patients: the pulmonary embolism response team (PERT). J Multidiscip Healthc. 2018;5;11:187-95.

2. Morrone D, Morrone V. Acute pulmonary embolism: focus on the clinical picture. Korean Circ J. 2018;48(7):661-3.

3. Kaya Z, Kayrak M, Gul EE, Altunbas G, Toker A, et al. The role of ischemia modified albumin in acute pulmonary embolism. Heart Views. 2014;15(4):106-10.

4. Inal ZO, Erdem SS, Gederet Y, Duran C, Kucukaydın Z, et al. The impact of serum adropin and ischemia modified albumin levels based on BMI in PCOS. Endokrynol Pol. 2018;69(2):13541.

5. Yamamoto T. Management of patients with highrisk pulmonary embolism: a narrative rewiew. J Intensive Care. 2018 Mar 2;6:16.

6. Torbicki A, Perrier A, Konstantinides S, Agnelli G, Galie N, et al. Guidelines on the diagnosis and management of acute pulmonary embolism: the task force for the diagnosis and management of acute pulmonary embolism of the European Society of Cardiology (ESC) Eur Heart J. 2008;29:2276-315.

7. Meyer G. Effective diagnosis and treatment of pulmonary embolism: Improving patient outcomes, Arch Cardiovasc Dis. 2014;107(67):406-14.

8. Waleed KB, Guan X, Li X, Yang Y, Wang Z, et al. Atrial fibrilation is related to lower incidence of deep venous thrombosis in patients with pulmonary embolism. J Thorac Dis. 2018;10(3):1476-82.

9. Noschang J, Guimarães MD, Teixeira DFD, Braga JCD, Hochhegger B, et al. Pulmonary thromboembolism: new diagnostic imaging techniques. Radiol Bras. 2018;51(3):178-86.

10. Raji H, JavadMoosavi SA, Dastoorpoor $M$, Mohamadipour Z, Mousavi Ghanavati SP. Overuse and underuse of pulmonary CT angiography in patients with suspected pulmonary embolism. Med J Islam Repub Iran. 2018:4;32:3.

11. Bozic J, Borovac JA, Galic T, Kurir TT, SupeDomic D, et al. Adropin and Inflammation Biomarker Levels in Male Patients With Obstructive Sleep Apnea: A Link With Glucose Metabolism and Sleep Parameters, J Clin Sleep Med. 2018;15;14(7):1109-18.

12. Zhao LP, You T, Chan SP, Chen JC, Xu WT. Adropin is associated with hyperhomocysteine and coronary atherosclerosis. Exp Ther Med. 2016;11(3):1065-70.

13. Hu W, Chen L. Association of Serum Adropin Concentrations with Diabetic Nephropathy. Mediators Inflamm. 2016;2016:6038261.

14. Lian W, Gu X, Qin Y, Zheng X. Elevated plasma levels of adropin in heart failure patients. Intern Med. 2011;50(15):1523-7.

15. Kumar KG, Trevaskis JL, Lam DD, Sutton GM, Koza RA, et al. Identification of adropin as a secreted factor linking dietary macronutrient intake with energy homeostasis and lipid metabolism. Cell Metab. 2008;8(6):468-81.

16. Aydin S, Kuloglu T, Aydin S, Kalayci M. Elevated adropin: a candidate diagnostic marker for myocardial infarction in conjunction with troponinI. Peptides. 2014;58:91-7.

17. Yosaee S, Soltani S, Sekhavati E, Jazayeri S. Adropin- A Novel Biomarker of Heart Disease: A Systematic Review Article. Iran J Public Health. 2016;45(12):1568-76.

18. Zhao LP, You T, Chan SP, Chen JC, Xu WT. Adropin is associated with hyperhomocysteine and coronary atherosclerosis. Exp Ther Med. 2016;11(3):1065-70.

19. Lovren F, Pan Y, Quan A, Singh KK, Shukla PC, et al. Adropin is a novel regulator of endothelial function. Circulation. 2010;14;122(11):185-92.

20. Liu MY, Ballard DW, Huang J, Rauchwerger AS, Reed ME, et al. Acute pulmonary embolism in emergency department patients despite therapeutic anticoagulation. West $\mathrm{J}$ Emerg Med. 2018;19(3):510-16.

21. Gök M, Kundi H, Kızıltunç E, Topcuoglu C, Ornek $\mathrm{E}$. The relationship between ischaemia-modified albumin and good coronary collateral circulation. Kardiol Pol. 2018;76(2):370-5.

22. Dogru M, Akoglu H, Kilinckaya MF, Ulfer G. Ischemia-modified albumin levels in children with asthma: a pilot study. Arch Argent Pediatr. 2018;1;116(4).

23. Güldoğan CE, Kılıç MÖ, Balamir İ, Tez M, Turhan T. Correlation between ischemia-modified albumin and Ranson score in acute pancreatitis. Ulus Travma Acil Cerrahi Derg. 2017;23(6):47276.

24. Özcan O, Erdal H, İlhan G, Demir D. Plasma Ischemia-Modified Albumin Levels and Dynamic Thiol/Disulfide Balance in Sickle Cell Disease: A Case-Control Study. Turk J Haematol. 2018;13;35(4):265-70. 
25. Wahab M. Ischemia modified albumin (IMA) in acute coronary syndrome (ACS) and left bundle branch block (LBBB). Does it make the difference?. Egypt Heart J. 2017;69(3):183-90.

26. Batmaz E, Edis EÇ, Eskiocak S, Hatipoğlu ON, Kaya S. The Diagnostic Value of Oxidative Stress Products in Pulmonary Embolism. Tur Toraks Der. 2011;12.3:100-4.

27. Kaya Z, Kayrak M, Gul EE, Altunbas G, Toker A, et al. The role of ischemia modified albumin in acute pulmonary embolism. Heart Views. 2014;15(4):106-10

28. Turedi S, Gunduz A, Mentese A, Karahan SC, Yilmaz SE, et al. Value of ischemia-modified albumin in the diagnosis of pulmonary embolism. Am J Emerg Med. 2007;25(7):770-3.

29. Turedi S, Gunduz A, Mentese A, Topbas M, Karahan SC, et al. The value of ischemiamodified albumin compared with d-dimer in the diagnosis of pulmonary embolism. Respir Res. 2008;30;9:49. 